\title{
Electrostatic Edge Instability of Lipid Membranes
}

\author{
M. D. Betterton ${ }^{1}$ and Michael P. Brenner ${ }^{2}$ \\ 1 Department of Physics, Harvard University, Cambridge, MA 02138 \\ 2 Department of Mathematics, MIT, Cambridge, MA 02139
}

This paper considers the effect of electrostatics on the stability of a charged membrane. We show that at low ionic strength and high surface charge density, repulsion between charges on the membrane renders it unstable to the formation of holes. A free straight edge is unstable to modulations with wavelength longer than the Debye screening length. Hence at low ionic strength, membranes will disintegrate into vesicles. We use these results to interpret stable holes in red blood cell ghosts (Steck et. al., Science 168:255(1970)).

Lipid membranes, the sheets which form the boundaries of cells, must maintain their integrity for cells to live [1]. Since a tear in a membrane exposes the hydrophobic interior of the sheet to water, tradition maintains that membranes have a high line tension (energy per unit length of exposed edge) and never spontaneously form holes. We show that membranes can form holes in a simple model of a charged membrane. By examining the competition between electrostatic repulsion and line tension, we find a parameter range where electrostatics dominates and membranes are unstable to hole formation. Although much previous work examines electrostatic effects on lipid assemblies [2] and the bending moduli of membranes [3, 4,5, w, we are only aware of one study addressing electrostatics and line tension [6].

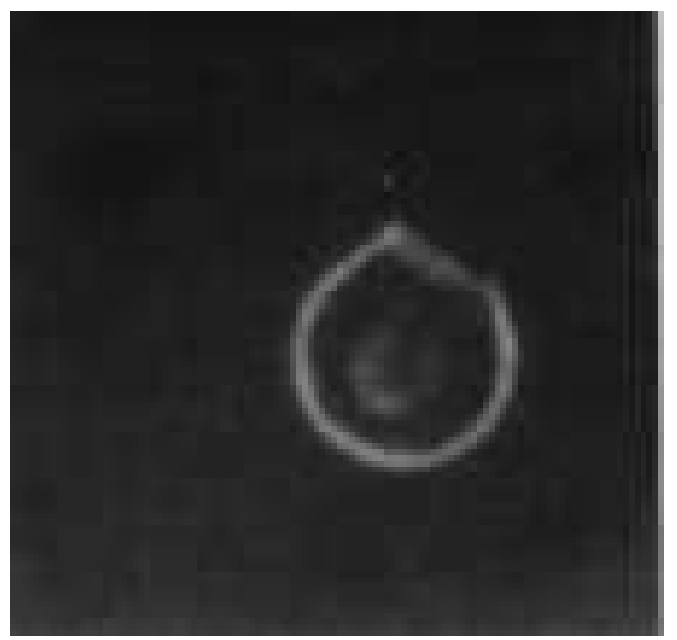

FIG. 1. Image of hole in red blood cell ghost, from Lieber and Steck [8] Figure 7.

These calculations were motivated by experiments which observed stable holes in red blood cell ghosts [7,8,9,9,10, the size of which depends on the ionic strength of the surrounding fluid [8] (Fig. 1). We compare our calculation with these experiments, and argue that the observed morphologies require the spectrin skeleton.

Consider a thin, axisymmetric membrane with constant charge density $\sigma$ on each side (Fig. 2a). The membrane attracts ions of the opposite sign, creating a screening layer (of thickness $\kappa^{-1}$ in Debye Huckel theory). We assume that the membrane is flat; this is a good approximation when the hole radius $R$ and screening length $\kappa^{-1}$ are small compared to the membrane radius of curvature. The plane of the membrane is at $z=0$. Throughout this paper, the membrane area and charge are held constant. 

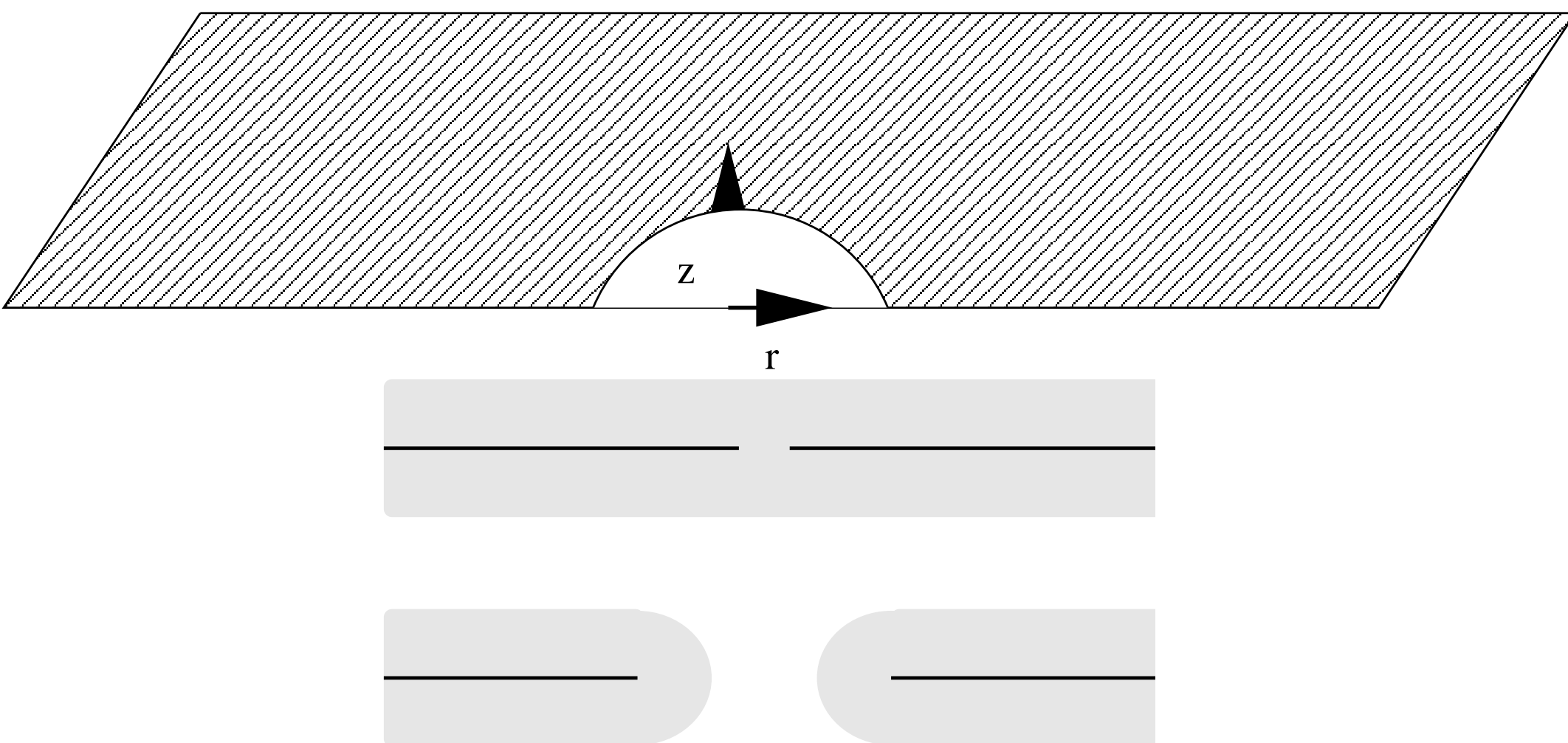

FIG. 2. (a) Sketch of a membrane hole; shading denotes constant charge density. (b) Sketch of the screening cloud (shaded) around the membrane (bold line). When $R<\kappa^{-1}$ (top), the screening cloud fills the hole. When $R>\kappa^{-1}$ (bottom), the cloud extends only $\kappa^{-1}$ from the edge.

Repulsion between charges on the membrane favors the creation and expansion of holes. Heuristically, the electrostatic energy is determined by the volume of the screening cloud: the total charge in the screening cloud is constant, so expanding the cloud lowers its energy. First, when the hole is small relative to a screening length (Fig. 2b), the cloud expands by a volume $\sim R^{2} \kappa^{-1}$ over the hole. Since the electric field strength is $\sim \sigma \epsilon_{w}^{-1}\left(\epsilon_{w}\right.$ is the dielectric constant of water), the energy decrease is $\sim \sigma^{2} \kappa^{-1} \epsilon_{w}^{-1} R^{2}$. The energy increases because an edge of membrane is exposed; if the membrane has a line tension $\gamma$, the energy cost of a small hole is $U \sim \gamma R-\sigma^{2} \kappa^{-1} \epsilon_{w}^{-1} R^{2}$. For small $R$ line tension dominates, and a hole closes.

In the opposite limit, the hole is much larger than a screening length. The cloud does not fill the hole, but inhabits an additional volume equal to that of a tube of radius $\kappa^{-1}$ and length $R$. The total energy is $U \sim\left(\gamma-\sigma^{2} \kappa^{-2} \epsilon_{w}^{-1}\right) R$. Depending on $\gamma, \sigma$, and $\kappa$, the energy can be positive or negative. The latter case corresponds to instability and hole growth.

We quantify this argument by calculating the energy of an idealized membrane hole. The relevant dimensionless parameter $P$ is the ratio of the line tension to the electrostatic energy per screening length of edge $P=\epsilon_{w} \kappa^{2} \gamma / \sigma^{2}$. We show that when line tension dominates, holes close. When $P \ll 1$, large holes grow. At intermediate $P \approx 2$ metastable holes exist due to the competition between energies. We then discuss the stability of a straight membrane edge to sinusoidal perturbations with wavenumber $q$. We show that at small $P$, and in the long wavelength limit $q \ll \kappa$, small perturbations lower the overall energy. In this regime, a flat membrane with a free edge will generically break into pieces of minimum size $O\left(\kappa^{-1}\right)$. The final section of the paper relates these results to the ghost experiments.

To compute the electrostatic energy of a hole, we use the linearized Poisson-Boltzmann equation [11] $\nabla^{2} \phi=\kappa^{2} \phi$. The boundary conditions are $\phi \rightarrow 0$ as $z \rightarrow \infty, \partial_{z} \phi(z=0)=-2 \pi \sigma / \epsilon_{w}, r>R$ and $\partial_{z} \phi(z=0)=0$ for $r<R$. The latter comes from symmetry of the potential about $z=0$. We Hankel transform to find

$$
\begin{aligned}
\phi(r, z) & =\frac{2 \pi \sigma e^{-\kappa z}}{\epsilon_{w} \kappa} \\
& -\frac{2 \pi \sigma R}{\epsilon_{w}} \int_{0}^{\infty} \frac{d k J_{0}(k r) J_{1}(k R)}{\sqrt{k^{2}+\kappa^{2}}} e^{-z \sqrt{k^{2}+\kappa^{2}}}
\end{aligned}
$$

where $J_{0}$ and $J_{1}$ are Bessel functions. Without a hole $(R=0)$, the potential reduces to that of an infinite charged sheet. 
The electrostatic energy is $U=2 \pi \int_{0}^{\infty} r d r \sigma \phi(z=0)$; we assume the total charge and the membrane area remain constant. The electrostatic contribution competes with the energy from line tension, $2 \pi \gamma R$. The energy change, nondimensionalized by $2 \pi \sigma^{2} /\left(\epsilon_{w} \kappa^{3}\right)$, depends on the parameter $P=\epsilon_{w} \kappa^{2} \gamma / \sigma^{2}$ and the dimensionless radius $\xi=\kappa R$

$$
\Delta U=P \xi-\pi \xi^{2}+2 \pi \xi^{3} \int_{0}^{\infty} \frac{d x J_{1}(x)^{2}}{x \sqrt{x^{2}+\xi^{2}}}
$$

When the hole is small $(\xi \ll 1)$, the integral in the third term is approximately $4 /(3 \pi)$, so the energy change of a hole is $\Delta U \approx P \xi-\pi \xi^{2}+8 / 3 \xi^{3}$. Thus, for small holes the line tension dominates over electrostatic effects. For larger $\xi$, the energy is shown in Fig. 3. When $P \gtrsim 2.2$, the energy increases monotonically as a function of $\xi$, so holes always close. For $2.0 \lesssim P \lesssim 2.2$, we find a local energy minimum with $\xi \sim 1$. In principle, holes in a membrane could be observed in this region of metastability. In practice, however, we believe that the range of parameters in which such holes exist is prohibitively narrow. When $P \lesssim 2$, the energy decreases linearly at large $\xi$, so that large holes will grow. In this regime there is an activation energy barrier to hole formation of size $\epsilon_{w} \kappa \gamma^{2} \sigma^{-2} / 2$.

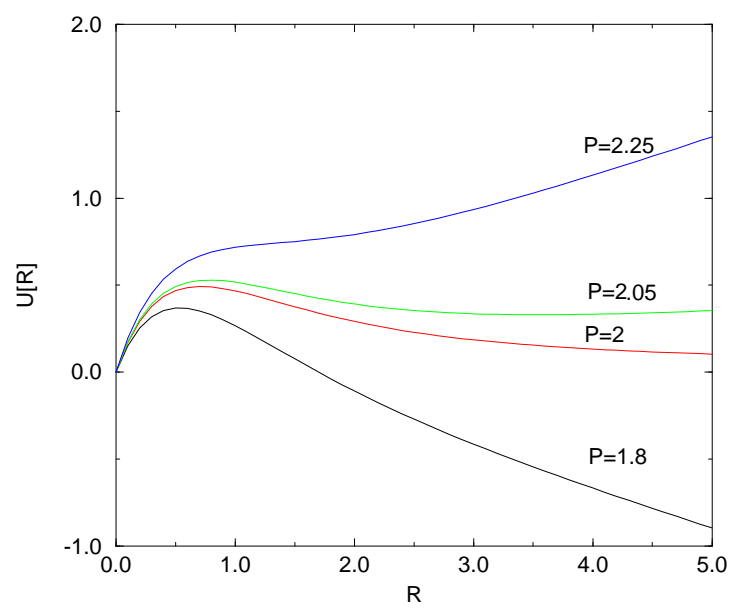

FIG. 3. Energy of a hole as a function of dimensionless radius $\kappa R$, for $P=1.8,2,2.05$ and 2.25. A typical barrier height is $10 \mathrm{kT}$ (for $P=1$, which corresponds to $n=1 \mathrm{mM}, \gamma=10^{-6} \mathrm{erg}$, and $\sigma=0.2 \mathrm{e} / \mathrm{nm}^{2}$ ).

A membrane with a growing hole ultimately has a long edge; is this edge stable? We consider a straight edge segment (at $x=0$ ) modulated by a sinusoidal perturbation $x=\epsilon \cos (q y)$. For this problem the charge density is $\sigma=\sigma_{0} \Theta(x+\epsilon \cos q y)$, where $\Theta$ is a step function. The energy follows from the linearized Poisson-Boltzmann approximation by expanding the potential and the charge density in powers of $\epsilon$. The first nonzero correction to the energy from the edge perturbation occurs at $O\left(\epsilon^{2}\right)$, and this change in the electrostatic energy (in dimensionless units) due to the edge modulation is $-\epsilon^{2} \kappa^{3} q^{-1} \ln \left(1+\kappa^{-2} q^{2}\right)$.

The perturbation increases the length of edge by $\Delta L=\pi / 2 \epsilon^{2} q$, resulting in an energy increase $\gamma \Delta L$ from line tension. For large $q$ line tension dominates, increasing the energy. For small $q \ll \kappa$, the total energy change is

$$
\Delta U=\frac{\epsilon^{2} q \kappa}{2}\left(\frac{P}{2}-1\right)+O\left(q^{2}\right),
$$

If (a) electrostatic effects dominate $(P<2)$ and $(b)$ the perturbation wavelength is greater than the screening length, modulations of the edge grow. The growth continues as long as the circumference of membrane fragments is longer than the screening length. When the fragment radius is of order $2 \pi \kappa^{-1}$ the instability will stop,and the fragment may close to form a vesicle. In the absence of charge, the fragment size must be $\gtrsim 10$ nm [15], so the energy associated with the free edge is larger than the bending energy required to close into a vesicle. With electrostatic effects included, it seems additional forces are necessary to promote vesicle formation. A candidate for red blood cells is spontaneous curvature: the higher charge density on the inner face of the bilayer [8] means the membrane prefers to bend towards the outer face [4]. Understanding the final vesicle sizes requires analyzing both the electrostatic edge instability and spontaneous curvature - in the Lew experiments, 99 spontaneous curvature effects play an important role in the experimental phenomenology. 
Observations of stable holes in red blood cell ghosts motivated this work 16]. Human red cells, if burst by osmotic stress and placed in a low-salt buffer, break into vesicles [7], which typically show the cytoplasmic (inner) side of the membrane facing outwards. Studies of this process [8,9] illustrate that vesiculation is preceded by the formation of stable holes in the membrane, whose size depends on the salt concentration of the solution and the membrane charge density. Lieber and Steck [8] showed that (a) the hole size increases with decreasing salt concentration; and (b) a decrease (increase) in the effective membrane charge density - via charged intercalators inserted into the membrane decreases (increases) the hole size. Our model qualitatively explains these trends, but uncertainties in our model and in experimentally measured parameters prevent precise numerical calculation. We have neglected (a) differences in charge density on the two sides of the membrane, (b) the insulating interior of the bilayer [4, and (c) nonlinear effects. Although there are experimental indications that some of these effects might be relevant [12], in the absence of quantitative information for the line tension and charge density, the simple model presented here is adequate.

Fig. 4 shows the stability boundaries as a function of the surface charge density $\sigma$ and the salt concentration $n$. We assume $\gamma=10^{-6}$ dyn, consistent with recent experiments 113. The phase boundaries follow $\sigma \propto n^{1 / 2}$. For typical membrane charge density $\sigma \sim 0.2 \mathrm{e} / \mathrm{nm}^{2}$, the crossover point $P \sim 2$ occurs at a salt concentration of $2 \mathrm{mM}$, which is in the range of the Lieber-Steck experiments. When the energy barrier for hole formation is of order a thermal energy $k_{B} T$, thermal fluctuations produce holes in the membrane. The dashed line in Fig. 4 shows the borderline for this instability [14]. Since the barrier scales like $\gamma^{2}$, the exact position of this important borderline is sensitive to the line tension $\gamma$.

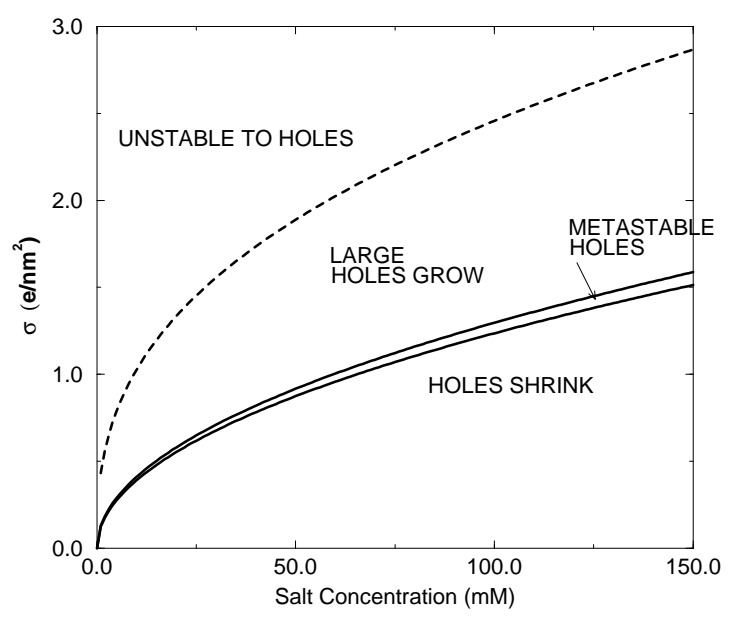

FIG. 4. Phase Diagram, depicting regimes of shrinking, growing and metastable holes, with $\gamma=10^{-6}$ dyn. The upper and lower solid lines depict $P=2$ and 2.2. The electrostatic edge instability is present everywhere above the solid lines. Note that the salt concentration (assuming a $1: 1$ salt such as $\mathrm{NaCl}$ ) is related to the Debye length via $\kappa^{2}=n / \ell^{2}$, where $\ell^{2}=\epsilon_{w} k_{B} T /\left(8 \pi e^{2} N\right)$, with $N$ Avogadro's number.

The electrostatic model qualitatively explains the dependence on salt concentration and membrane charge density in the experiments. However, electrostatic effects are not sufficient: the edge instability predicts that holes either grow indefinitely (resulting in vesiculation) or close completely. The observations [8,9] of stable holes for long time periods contradict this prediction and indicate that a stabilizing effect is necessary.

Several aspects of the experiments indicate that the spectrin skeleton - a protein mesh anchored to the membraneis this stabilizing element. The electron micrographs of Lew et. al. [9] show that the spectrin network is intact before vesiculation occurs, and as the ghosts disintegrate into vesicles the spectrin mesh detaches from the membrane. Lieber and Steck [8] found that proteins known to covalently cross-link spectrin stabilize the hole, preventing changes in radius as the ionic strength of the solution is changed. Conditions known to promote breakdown of spectrin (such as increase in temperature or digestion by enzymes [8]) cause the ghosts to vesiculate. If the spectrin provides the restoring force needed to stabilize the membrane, force balance implies $k_{s} R=-d U / d r$, where $k_{s}$ is the spectrin area expansion modulus and $U$ the hole energy computed above. At small $P$, we find

$$
R=\frac{4 \pi \sigma^{2} \ell^{2}}{\epsilon_{w} k_{s}} \frac{1}{n} .
$$

Figure 5 shows Lieber and Steck's data [8], replotted on a double logarithmic scale. 


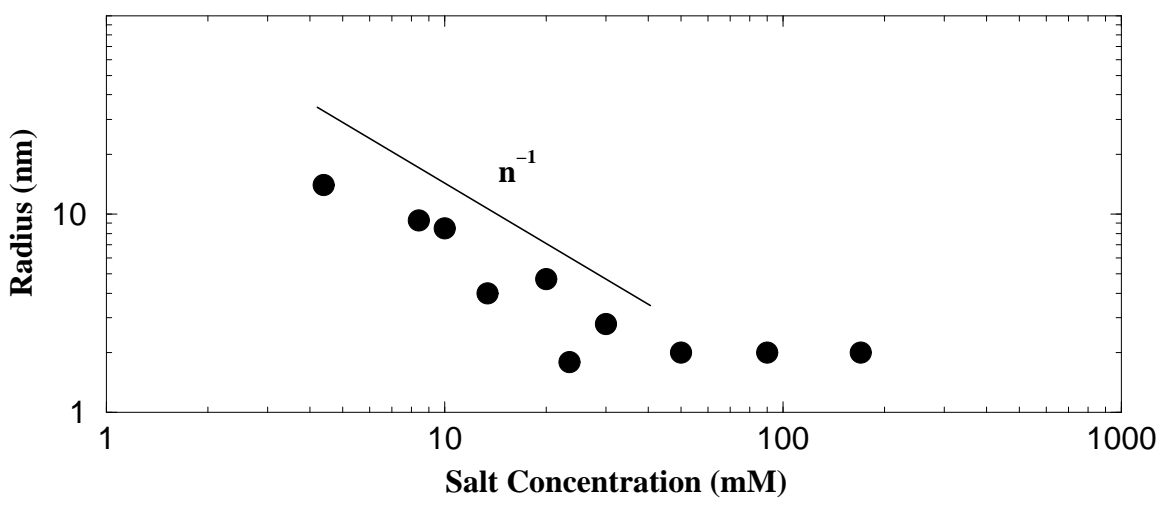

FIG. 5. Radius of hole versus salt concentration from Ref. [8]. The solid line is $R \sim n^{-1}$.

The data obey this law over a decade in hole size. To check the consistency we fit the prefactor of the scaling law. A best fit gives the prefactor $c=7.0 \cdot 10^{-6} \mathrm{~cm} \mathrm{M}$. For $\sigma$ in units of $e / \mathrm{nm}^{2}$ and $k_{s}$ in dyn, we find $\sigma^{2} / k_{s}=0.02$. This ratio is consistent with the charge density $\sigma=10^{-2}$ and bulk modulus $k_{s}=10^{-2}$ from independent measurements [17].

The mechanism of vesiculation by electrostatic edge instability does not require spontaneous curvature; hence it differs from other explanations of vesiculation 18,3, 1 . There are conditions under which vesiculation appears to be driven by spontaneous curvature [19]. It seems likely that both mechanisms for vesiculation operate in practice.

For physiological conditions, membrane charge densities range from -0.03 to $-0.24|e| / \mathrm{nm}^{2}$ [4, 20, 21] and $\mathrm{NaCl}$ concentrations are around 150mM. The phase diagram Fig. 4 predicts that such membranes should be stable, although conditions in vivo are within an order of magnitude of the stability boundary. This indicates that the edge instability could be important in living systems. Partition of the nuclear envelope (the membrane surrounding the nucleus) during cell division provides a possible example [22,23]. Because it is a double bilayer [1], its effective line tension is much smaller than a typical membrane. The qualitative behavior of the nuclear envelope is similar to the phenomenology described here: during most of the cell cycle, the nuclear envelope is attached to a protein meshwork called the nuclear lamina [22]. Near the start of cell division, the lamina disassembles - and the nuclear envelope is observed to fill with holes and vesiculate [23]. The many vesicles are divided between the two daughter cells during division.

Acknowledgements: We are grateful to Professor Ted Steck, for patiently introducing us to his experiments, and David Lubensky for useful discussions. MPB acknowledges support from the A.P. Sloan foundation. MDB acknowledges support by a Fellowship from the Program in Mathematics and Molecular Biology at the Florida State University, with funding from the Burroughs Wellcome Fund Interfaces Program.

[1] B. Albert et al., Molecular Biology of the Cell (Garland Publishing, Inc., New York, 1989).

[2] J. Israelachvili, D. Mitchell, and B. Ninham, J. Chem. Faraday Trans. 2 72, 1525 (1976)

[3] M. Winterhalter and W. Helfrich, J. Phys. Chem. 92, 6865 (1988). M. Winterhalter and W. Helfrich, J. Phys. Chem. 92, 327 (1992).

[4] T. Chou, M. Jaric, and E. D. Siggia, Biophys. J. 72, 2042 (1997).

[5] B. Duplantier et. al. Phys. Rev. Lett. 65, 508 (1990).

[6] A.G. Petrov et. al. Adv. Liq. Cryst. Research (L. Bata, ed), 2695 (1980). This paper briefly discusses line tension in an external electric field.

[7] T. Steck et. al. Science 168, 255 (1970); T. Steck and J. Kant, Meth. Enzym. 31, 172(1974).

[8] M. R. Lieber and T. L. Steck, J. Bio. Chem 57, 11651 (1982). M. R. Lieber and T. L. Steck, J. Bio. Chem 57, 11660 (1982).

[9] V. Lew et. al. J. Cell Biol. 106, 1893 (1988).

[10] V. L. Lew, S. Muallem, and C. A. Seymour, Nature 296, 742 (1982).

[11] The linearized approximation is valid whenever $e \phi \ll k_{B} T$. For physiological salt concentrations, this condition holds, but a crossover occurs at lower salt concentration; $e \phi \sim k_{B} T$ for $25 \mathrm{mM}$ salt.

[12] An example of a nontrivial electrostatic effect observed in the experiments [8] is that varying the concentration of divalent cations has a much larger effect than would be anticipated from changing the Debye length.

[13] D. V. Zhelev and D. Needham, Biochemica et Biophysica Acta 1147, 89 (1993). 
[14] The dashed curves in Fig. 4 occur outside the regime where linearized Poisson-Boltzmann is valid. The exact solution of the Poisson-Boltzmann equation for an infinite plane indicates that nonlinearity leads to stronger confinement of the screening cloud (effectively decreasing the screening length). Nonlinearities do not change the scaling of the electrostatic energy with the hole radius $R$, and therefore do not qualitatively affect the results.

[15] W. Helfrich, Phys. Lett. 50A, 115 (1974); P. Fromherz, Chem. Phys. Lett. 94, 259 (1983).

[16] More recent works focus on holes in membranes for other reasons. See [13]; J. Moroz and P. Nelson, Biophys. J. 72, 2211-16 (1997); R. Bar-Ziv et. al. Phys. Rev. Letter 75, 3481 (1995). W. Sung and P.J. Park, Biophys. J. 731797 (1997).

[17] H. Engelhardt and E. Sackmann, Biophys. J. 54, 495 (1988); R. Waugh and E. Evans, Biophys. J. 26, 115(1979); D. Discher et. al. Science 266,1032 (1994); Y. Kantor and D. R. Nelson, Phys. Rev. A.36 4020(1987).

[18] H. Hauser, Proc. Nat. Acad.Sci 86, 5251 (1989).

[19] W. Li and T. H. Haines, Biochemistry 25, 7477 (1986).

[20] W. Chow and J. Barber, J. Biochem. Biophys. Methods 3, 173 (1980).

[21] F. Sack, D.Priestley, and A. Leopold, Planta 157, 511 (1983).

[22] G. Warren, Ann. Rev. Biochem. 323 (1993).

[23] U. Roos, Chromosoma 40, 43 (1973). 